\title{
Risk of Neoplastic Squall Downstream Cytokine Storm of COVID-19 in Male Patients
}

\author{
(1) Levent Türkeri ${ }^{1}$, (1) Ercüment Ovalı² \\ ${ }^{1}$ M.A. Aydınlar Acıbadem University School of Medicine, Departments of Urology, Istanbul, Turkey \\ 2 M.A. Aydinlar Acıbadem University School of Medicine, Hematology and Immunology, Istanbul, Turkey
}

\section{Introduction}

Scientific information about the molecular mechanisms of Severe Acute Respiratory syndrome coronavirus-2 (SARS-CoV-2) viral infection reveals crossing paths with prostate carcinogenesis through induction of recurrent TMPRSS-ERG gene fusion in an inflammatory microenvironment. Therefore, possibility of a subsequent increased risk of prostate cancer after this viral pandemic requires proper attention with further research.

A novel betacoronavirus identified as SARS-CoV-2 is the cause of a current global public health crisis. The resulting disease was termed coronavirus disease-2019 (COVID-19) with a clinical presentation ranging over a wide spectrum (1). A subgroup of patients develop severe and potentially lethal disease associated with an immune profile characterised by high levels of proinflammatory cytokines suggesting a cytokine storm $(1,2)$. Recent studies identified the molecular pathways of this viral infection in which SARS-CoV-2 enters host cells after binding viral spike (S) surface envelope proteins to cellular angiotensinconverting enzyme 2 (ACE2) receptors and proteolytic S protein priming by TMPRSS2, which is a transmembrane serine protease (3). Therefore, modulation of TMPRSS2 expression as well as cytokine receptor blockade have been suggested as promising potential treatment strategies against COVID-19 $(1,4)$. Yet the pivotal role of ACE2 and TMPRSS2 in COVID-19 may rather have more ominious implications for men.

\section{Role of TMPRSS2 and Inflammation in Prostate Cancer}

TMPRSS2 is an androgen responsive, highly expressed gene in prostatic tissue and strongly associated with prostatic carcinogenesis via fusion with oncogene ETS family transcription factors, mainly ERG (5). Recently, a significant role of inflammation leading to recurrent TMPRSS2-ERG gene fusions has been demonstrated via induction of DNA breaks in an inflammatory environment (6). In addition to previous studies indicating an etiologic link between inflammation and prostate carcinogenesis, a recent population based prospective cohort study provided further evidence supporting the role of inflammation in prostate carcinogenesis and progression $(7,8)$. The Prostate Cancer Study throughout life (PROCA-life), demonstrated an increased risk of prostate cancer with a much higher risk of metastatic disease in men with high systemic inflammatory scores (8).

\section{Possible Mutual Mechanisms}

Both ACE2 and TMPRSS2 which are the key molecules for pathogenicity facilitating SARS-CoV-2 viral entry in to the host cells are highly expressed in urogenital organs, especially in prostate (see Related links) and render them susceptible to damage by this virus as well as accompanying inflammation $(5,9)$. Thus potential inherent tropism of SARS-CoV-2 for prostate and associated local and/or systemic inflammation with high levels of cytokines in this disease provide an extremely convenient microenvironment for carcinogenesis through induction of recurrent TMPRSS2-ERG gene fusions. Although significant injury in renal and testicular tissue was reported, information concerning histopathological findings of prostate tissue associated with SARS-CoV-2 infection is still missing (9). Also, incidence of lower urinary tract symptoms which may be associated with severe prostatic inflammation is currently not clear. This may in part related to clinical presentation of the disease where predominant symptoms are pulmonary in origin and often life threatening in these patients which probably sideline and obscure any symptoms related to lower urinary tract. Asymptomatic prostatic inflammation is also a possibility which is in fact the case in many patients (10). 


\section{Conclusions}

Available scientific evidence raises a significant concern about possibility of increased risk of prostate cancer development by recruiting the same key molecules responsible for carcinogenesis as well as SARS-CoV-2 viral entry in to host cells triggering immune response and subsequent inflammatory cascade with downstream severe inflammatory microenvironment at target organs. Therefore, the medical community should tailor the follow-up of recovering male patients accordingly until this hypothesis is refuted properly by further research.

\section{Authorship Contributions}

Authors contributed equally to this work.

Conflict of Interest: No conflict of interest was declared by the authors.

Financial Disclosure: No financial disclosure was declared by the authors.

\section{References}

1. Mehta P, McAuley DF, Brown M, et al. COVID-19: consider cytokine storm syndromes and immunosuppression. Lancet 2020;395:1033-1034.

2. Huang $C$, Wang Y, Li X, et al. Clinical features of patients infected with 2019 novel coronavirus in Wuhan, China. Lancet 2020;395:497-506.

3. Hoffmann M, Kleine-Weber H, Schroeder S, et al. SARS-CoV-2 Cell Entry Depends on ACE2 and TMPRSS2 and Is Blocked by a Clinically Proven Protease Inhibitor. Cell 2020;181:271-280.
4. Stopsack KH, Mucci LA, Antonarakis ES, Nelson PS, Kantoff PW. TMPRSS2 and COVID-19: Serendipity or Opportunity for Intervention? Cancer Discov 2020;10:779-782.

5. Chen YW, Lee MS, Lucht A, et al. TMPRSS2, a serine protease expressed in the prostate on the apical surface of luminal epithelial cells and released into semen in prostasomes, is misregulated in prostate cancer cells. Am J Pathol 2010;176:2986-2996.

6. Mani RS, Amin MA, Li X, et al. Inflammation-Induced oxidative stress mediates gene fusion formation in prostate cancer. Cell Rep 2016;17:2620-2631.

7. Gurel B, Lucia MS, Thompson IM Jr, et al. Chronic inflammation in benign prostate tissue is associated with high-grade prostate cancer in the placebo arm of the prostate cancer prevention trial. Cancer Epidemiol Biomarkers Prev 2014;23:847-856.

8. Stikbakke $E$, Richardsen $E$, Knutsen $T$, et al. Inflammatory serum markers and risk and severity of prostate cancer: The PROCA-life study. Int J Cancer 2020;147:84-92.

9. Wang $S$, Zhou $X$, Zhang $T$, Wang $Z$. The need for urogenital tract monitoring in COVID-19. Nat Rev Urol 2020;17:314-315.

10. Sfanos KS, Isaacs WB, De Marzo AM. Infections and inflammation in prostate cancer. Am J Clin Exp Urol 2013;1:3-11.

\section{Related Links}

The Human Protein Atlas portal (ACE): https://www.proteinatlas. org/ENSG00000159640-ACE

The Human Protein Atlas portal (TMPRSS2): https://www. proteinatlas.org/ENSG00000184012-TMPRSS2 